\title{
ChemComm
}

Check for updates

Cite this: Chem. Commun., 2021, 57, 7645

Received 24th May 2021, Accepted 28th June 2021

DOI: $10.1039 / \mathrm{d} 1 \mathrm{cc} 02722 \mathrm{k}$

rsc.li/chemcomm

\section{Facile protein conjugation of platinum for light-activated cytotoxic payload release $\dagger$}

\author{
Cinzia Imberti, (D) *a Frederik Lermyte, (D) ab Emily P. Friar, (D) ${ }^{\text {a }}$ Peter B. O'Connor (D) ${ }^{a}$ \\ and Peter J. Sadler (D)*a
}

The novel Pt(Iv) complex trans, trans- $\left[\mathrm{Pt}\left(\mathrm{N}_{3}\right)_{2}(\mathrm{Py})_{2}(\mathrm{OH})\left(\mathrm{OCO}-(\mathrm{PEG})_{2}-\right.\right.$ NHCSNH-Ph-NCS)] (Pt4) conjugates to the side chain of lysine amino acids in proteins under mild conditions. Reaction with myoglobin generated a bioconjugate that was stable in the dark, but released a $\mathrm{Pt}(\mathrm{IV})$ prodrug upon visible light irradiation. A similar procedure was used to conjugate Pt4 to the antibody trastuzumab, resulting in the first photoactivatable $\mathrm{Pt}(\mathrm{Iv})$-antibody conjugate, demonstrating potential for highly selective cancer phototherapy.

Despite their widespread clinical use, platinum(II) anticancer drugs often present severe side effects, which critically affect patients' quality of life. The use of relatively inert Pt(Iv) prodrugs that can undergo intracellular reduction to cytotoxic Pt(II) species in cancer cells has been widely investigated to minimise systemic toxicity. ${ }^{1}$ However, non-tumour specific reduction limits the benefits of these agents. No Pt(Iv) chemotherapeutics have been clinically approved to date.

Another way to minimise the systemic toxicity of a chemotherapeutic is to increase its cancer-targeting capability using a wide range of delivery vectors (e.g. liposomes, nanotubes, peptides, hormones, and proteins). ${ }^{1} \mathrm{~A}$ few Pt-protein conjugates have been reported so far. These include albumin adducts bound to octahedral $\mathrm{Pt}(\mathrm{Iv})$ via non-covalent interactions with fatty acid-like axial ligands, ${ }^{2}$ or via a maleimide group targeting the free cysteine residue (Cys34) of albumin., A few Pt-antibody conjugates have also been developed, mainly involving Pt(II) agents, but these have not displayed potential for clinical translation. ${ }^{5-8}$ Development of Pt-protein adducts presents several challenges, including the need for mild, watercompatible conjugation procedures that do not excessively disrupt the protein structure. Release of the cytotoxic payload

\footnotetext{
${ }^{a}$ Department of Chemistry, University of Warwick, Gibbet Hill Road,

CoventryCV4 7AL, UK. E-mail: cinzia.imberti@warwick.ac.uk,

P.J.Sadler@warwick.ac.uk

${ }^{b}$ Department of Chemistry, Technical University of Darmstadt,

Alarich-Weiss-Strasse 4, Darmstadt 64287, Germany

$\dagger$ Electronic supplementary information (ESI) available. See DOI: 10.1039/ d1 1 c $02722 k$
}

from the protein vector once the tumour target has been reached is also important to ensure efficacy.

Unlike traditional $\mathrm{Pt}(\mathrm{Iv})$ prodrugs, photoactivatable $\mathrm{Pt}(\mathrm{Iv})$ azido complexes are inert and non-toxic in the presence of bio-reductants, but release cytotoxic Pt(II) species upon irradiation with visible light, providing spatial and temporal control of their chemotherapeutic activity. ${ }^{9}$ Derivatisation of these complexes can provide, for example, cancer-targeting peptide conjugates. ${ }^{10,11}$ A protein adduct has also been synthesised, which exploits a pendant biotin moiety to attach the Pt(Iv) agent to avidin, utilising the high biotin-avidin affinity. ${ }^{12}$

In this work, the novel photoactivatable $\mathrm{Pt}(\mathrm{Iv})$ derivative trans,trans-[Pt $\left.\left(\mathrm{N}_{3}\right)_{2}(\mathrm{Py})_{2}(\mathrm{OH})\left(\mathrm{OCO}-(\mathrm{PEG})_{2}-\mathrm{NHCSNH}-\mathrm{Ph}-\mathrm{NCS}\right)\right]$ (Pt4, Fig. 1) has been synthesised with a pendant isothiocyanate group, as a platform for facile, universal attachment to proteins via lysine side chain conjugation.

Pt4 was synthesised in 3 steps from the prototype complex Pt1. First a Fmoc-protected PEG linker was introduced to enhance biocompatibility and water-solubility of the agent and to act as a spacer between Pt and amino acid residues with the aim of minimising unwanted Pt/protein interactions. Then, upon deprotection of the primary amine function, the resulting intermediate $\mathbf{P t} 3$ was coupled using a large excess of phenyl bisisothiocyanate to yield a 1:1 Pt-isothiocyanate derivative Pt4. The identity and the purity of Pt4 were confirmed by NMR, reversed-phase HPLC, and HR-tandem mass spectrometry (see ESI $\dagger$ ). Both Pt4 and its precursors displayed very high dark stability, but were photoactivated by irradiation with blue light $(420 \mathrm{~nm})$, as was evident from the reduction in intensity of the Pt $\leftarrow \mathrm{N}_{3}$ LMCT band in the UV-vis spectrum (Fig. S1-S3, ESI $\dagger$ ).

Myoglobin was used as a model protein to evaluate the ease of protein conjugation of Pt4 and investigate the properties of the resulting conjugate. Myoglobin (equine, $M_{\mathrm{W}} 17.6 \mathrm{kDa}$, 153 amino acids, Fig. 1) is a small protein carrying a noncovalently bound haem group, and 10 of its 19 Lys residues are solvent-accessible (based on the crystal structure). ${ }^{13}$ Furthermore, its Soret absorption band $(c a .408 \mathrm{~nm})$ is well separated 


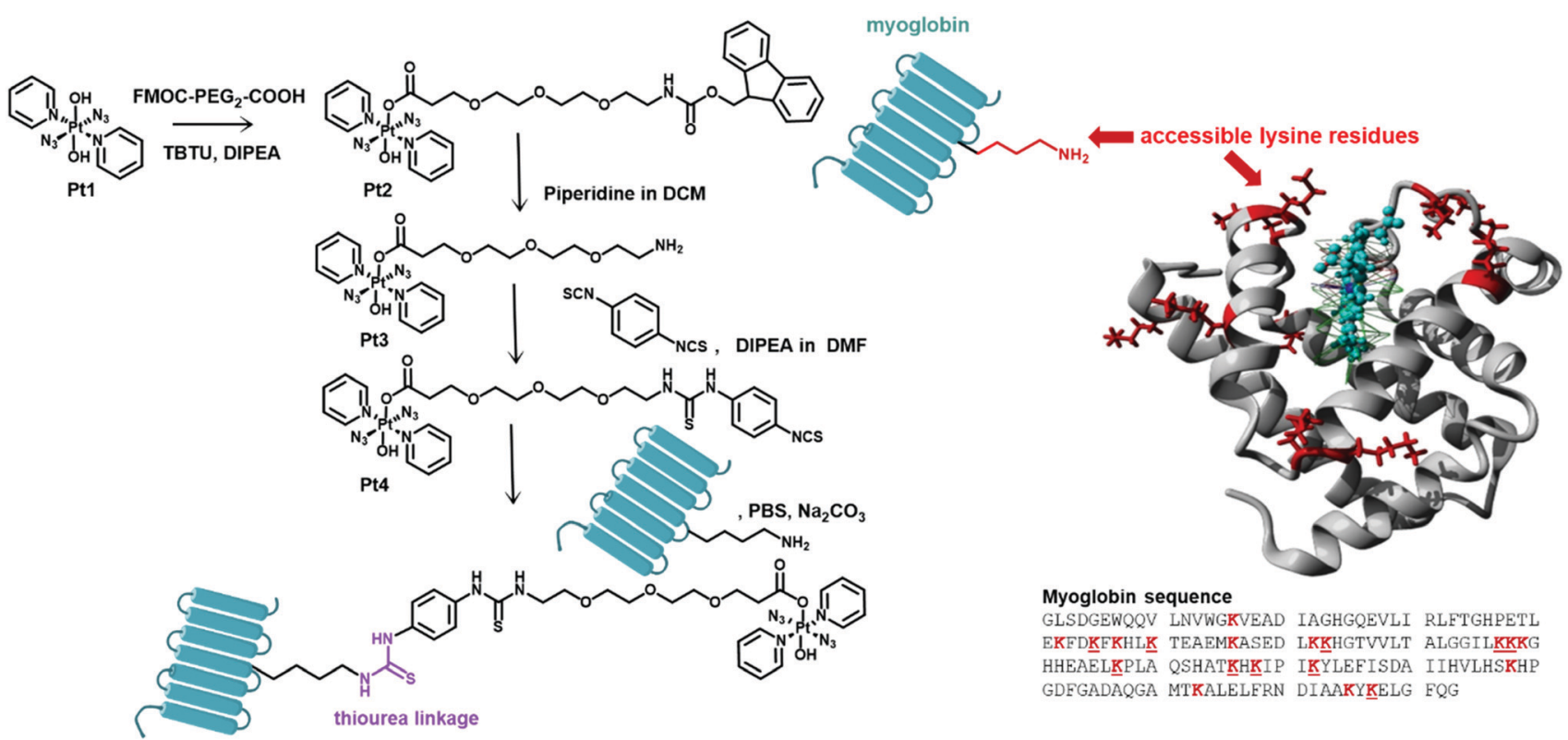

Fig. 1 Synthetic route to Pt4 and its myoglobin derivative myo-Pt4 (left). Structure and sequence of myoglobin highlighting accessible lysine residues (underlined in structure) based on the X-ray crystal structure of equine myoglobin (right, PDB accession code 1WLA).

from the LMCT band of the Pt(Iv) azido complex (ca. $288 \mathrm{~nm})$, enabling precise quantification of protein concentration within the bioconjugate solution.

Myoglobin was reacted with 5 mol equiv. Pt4 in water at $\mathrm{pH}$ 8.5-9 for $4 \mathrm{~h}$ followed by gel filtration to remove unreacted Pt4. HPLC analysis of the mixture was performed to verify successful conjugation and absence of unreacted Pt4 using a size-exclusion (4-400 kDa) column and a diode-array detector (DAD). The HPLC chromatograms provided a straightforward differentiation between the unmodified protein and the $\mathrm{Pt}(\mathrm{Iv})$ conjugate myo-Pt4, owing to their different UV-vis absorption spectra (Fig. 2A). Unmodified myoglobin, for which $A_{360}>A_{254}$ and $\Delta A=A_{254}-A_{360}<0$, gave a sharp negative HPLC peak at 3 min 45 s. In contrast, myo-Pt4 displayed one peak at a similar retention time to myoglobin $(4 \min 4 \mathrm{~s}$ ) but positive $\Delta A$, suggesting that the new chemical entity was similar in size to myoglobin, but had a different UV-vis spectrum. Notably, a broad positive peak at $6 \mathrm{~min} 33 \mathrm{~s}$ was recorded for Pt4 (Fig. S5, ESI $\dagger$ ). DAD spectra for each HPLC peak (Fig. 2A and Fig. S5, ESI $\dagger$ insets), show that myo-Pt4 displays the peaks
(A)

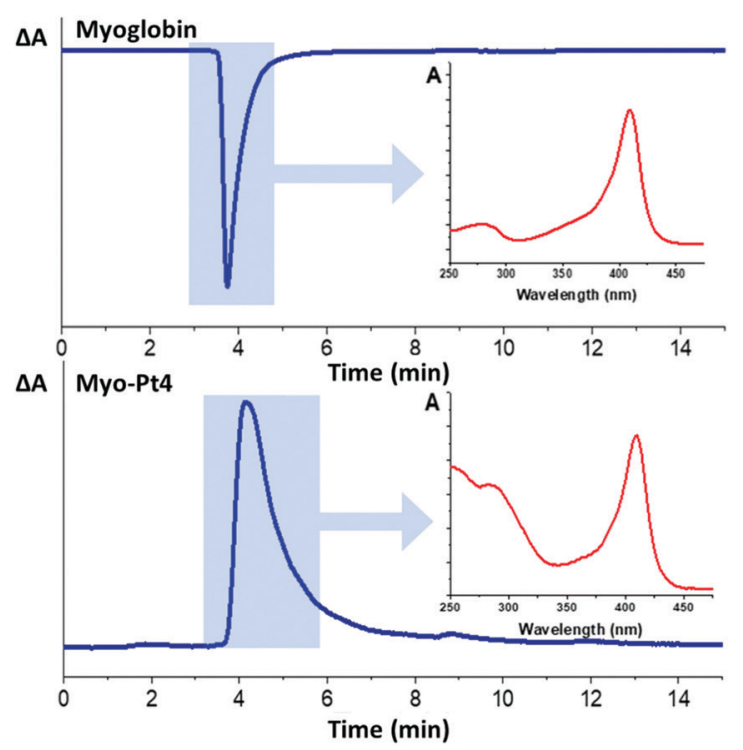

(B)

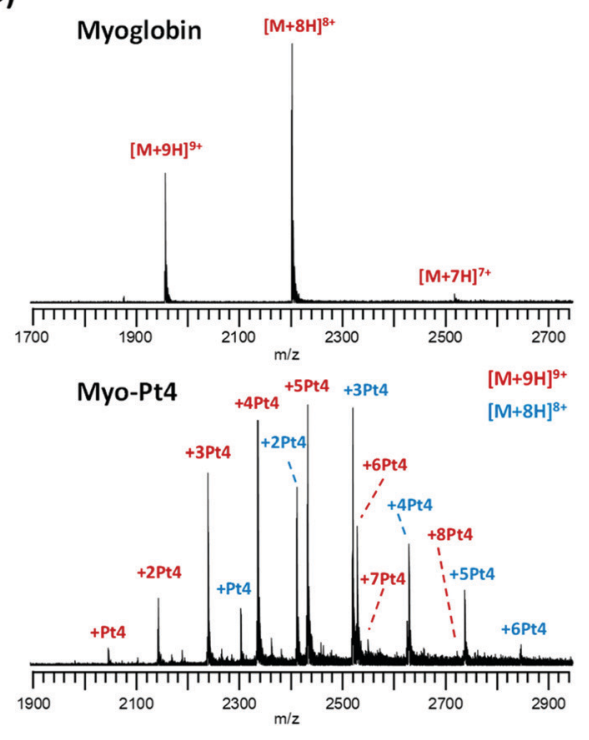

Fig. 2 Characterisation of myo-Pt4 using (A) HPLC (detection $\lambda: 254 \mathrm{~nm}$, reference: $360 \mathrm{~nm}, \Delta A=A_{254}-A_{360}$ ), insets show DAD spectrum for the peak, and (B) FTICR-MS comparing native holo-myoglobin (top panel) with the bioconjugate (bottom panel). 
corresponding to both the myoglobin haem group (408 $\mathrm{nm}$ ) and Pt4 (254 nm, $288 \mathrm{~nm}$ ). An average Pt4/myoglobin ratio of 5.6 was determined for myo-Pt4 using UV-vis spectroscopy to measure protein concentration from the absorbance of the Soret band ([myoglobin $]=2.9 \mu \mathrm{M})$ and ICP-MS to determine Pt4 concentration $([\mathrm{Pt}]=16.4 \mu \mathrm{M})$.

ESI-MS analysis of the mixture identified different myoglobin-Pt4 conjugates containing up to $8 \mathbf{P t 4}$ complexes per myoglobin molecule, while no unmodified myoglobin remained (Fig. 2B). Importantly, no apo-myoglobin was observed, demonstrating retention of the haem group throughout the conjugation/purification process and the MS analysis. This is particularly significant as it highlights the gentle reaction and MS conditions employed. Analysis of the relative intensity of the peaks for the most prominent charge state (9+) also allowed calculation of an approximate average Pt/protein ratio of $4.4 \mathrm{Pt} 4 /$ myoglobin in good agreement with the value obtained by UV-vis/ICP-MS measurements (5.6).

Myo-Pt4 was stable in the dark with no changes in UV-vis absorbance over $2 \mathrm{~h}$, showing that the Pt(Iv) azido complex retains its stability when conjugated to myoglobin (Fig. 3).

In contrast, upon irradiation with blue light both the $288 \mathrm{~nm}$ peak (Pt $\leftarrow \mathrm{N}_{3}$ LMCT band) and the $408 \mathrm{~nm}$ peak (myoglobin Soret band) decreased significantly indicating photodecomposition (Fig. 3). Interestingly, no photodecomposition was observed when native myoglobin underwent the same treatment (Fig. S6, ESI $\dagger$ ) suggesting that the haem group may interact with Pt4 during photodecomposition. Further investigation is required to confirm this possibility.

Notably, photoactivation was also observed upon green light irradiation ( $520 \mathrm{~nm}, 15 \%$ decrease in $A_{288}$ peak at $2 \mathrm{~h}$ Fig. S7, ESI $\dagger$ ), although to a lesser extent compared to blue light (30\% decrease). Size-exclusion HPLC of the irradiated mixture revealed the presence of several peaks (Fig. S8, ESI $\dagger$ ), but MS analysis of the sample was complicated by the complex nature of the mixture. This was unsurprising since a high ratio of $\mathrm{Pt} /$ protein, although advantageous to guarantee sufficient cytotoxic payload delivery, complicates the characterisation of the conjugate. Therefore, to gain more insight into the nature of the myo-Pt4 adducts and the photochemical reactions that take place upon photoactivation, the conjugation was repeated using only 1 mol equiv. of Pt4, with the aim of selectively forming a 1:1 adduct. MS analysis revealed the presence of unmodified myoglobin in the sample as well as the $1: 1$
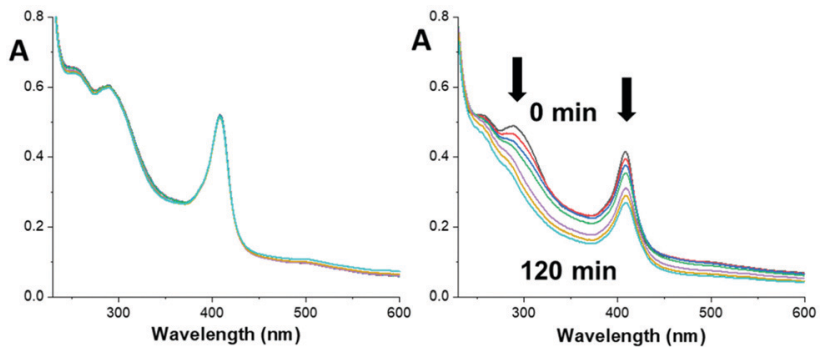

Fig. 3 UV-vis spectra of myo-Pt4 over $2 \mathrm{~h}$ in the dark (left) or upon $420 \mathrm{~nm}$ light irradiation (right), showing absorbance reduction at $288 \mathrm{~nm}$ and $408 \mathrm{~nm}$ (myoglobin Soret band). conjugate (Fig. S9A, ESI $\dagger$ ). Notably, tandem MS analysis of the $1: 1$ conjugate with ECD produced unmodified fragments spanning the entire myoglobin sequence (Table S1, ESI $\dagger$ ), suggesting that multiple accessible lysines are susceptible to modification by compound Pt4, consistent with myoglobin's $\mathrm{X}$-ray structure (Fig. 1). MS investigation of this new sample after $2 \mathrm{~h}$ irradiation with blue light (Fig. S9B, ESI $\dagger$ ) showed no intact conjugate, but two main peaks were identified with masses of $17706.096 \mathrm{Da}$ attributable to loss of $\left[2 \times \mathrm{N}_{3}{ }^{\bullet}\right.$ and $\mathrm{OH}$ ] (calc. mass $=17706.114 \mathrm{Da}$ ) and $17353.073 \mathrm{Da}$ to loss of $\left[2 \times\left(\mathrm{C}_{5} \mathrm{H}_{5} \mathrm{~N}\right), 2 \times\left(\mathrm{N}_{3}\right), \mathrm{OH}\right.$ and Pt] (calc. mass $=17353.065 \mathrm{Da}$ ). The first peak showed typical photodecomposition patterns observed previously for the photoactivation of compound Pt1, with loss of the azido ligands and an $\mathrm{OH}$ group. In contrast, the second peak demonstrates how the platinum complex is at least partially released from the protein upon light activation. Importantly, the complex released upon irradiation is the prototype complex Pt1 that can then undergo its own photodecomposition pathways, as previously investigated, ${ }^{14}$ leaving the PEG isothiocyanate linker bound to the protein.

Encouraged by these results for myo-Pt4, we investigated Pt4 conjugation to antibodies, which are sensitive to the solution environment and can easily precipitate, form aggregates, or denature when manipulated at $\mathrm{pH}$ and concentrations of salts that differ from physiological conditions.

As a proof of concept, we investigated conjugation to the Her2-targeting monoclonal antibody trastuzumab (Herceptin ${ }^{\circledR}$, $M_{\mathrm{W}} \approx 148 \mathrm{kDa}$ ) used in the treatment of Her2-positive breast and gastric cancers. ${ }^{15}$ In addition to being clinically relevant, trastuzumab can form stable antibody-drug-conjugates (ADCs) with chemotherapeutics via its lysine residues, as shown in the clinically approved ADC with the microtubule inhibitor maytansine (trastuzumab emtansine). ${ }^{16}$

Trastuzumab was reacted with 5 mol equiv. Pt4 in PBS at $\mathrm{pH} \approx 8.5$ for $2 \mathrm{~h}$, followed by purification using ultracentrifugation. As expected, ESI-MS analysis of trastuzumab-Pt4 (Fig. 4) revealed a more complex pattern of modification compared to myo-Pt4 owing to the higher $M_{\mathrm{W}}$, as well as heterogeneity of trastuzumab in terms of glycosylation and other posttranslational modifications. ${ }^{17}$ Notably, while peaks for unmodified trastuzumab were still visible in the mixture, the presence of Pt4:trastuzumab adducts was evident. Due to the aforementioned heterogeneity, the peaks for the unmodified antibody possess an intrinsic width of around $900 \mathrm{Da},{ }^{17}$ i.e., on the order of the mass of Pt4. As a result, it is fundamentally impossible to baseline-separate the different adducts as for myoglobin. Specifically, the most heavily glycosylated portion of the Pt-free population overlaps with the least glycosylated portion of the 1:1 Pt: antibody adduct, and similarly for other adducts (see Fig. 4). To overcome the limitations due to the broad trastuzumab peaks, we simulated spectra of trastuzumab with zero, one, and two additions of Pt4 (Apo, 1 Pt4, 2 Pt4 in Fig. 4, respectively). A linear combination (blue dashed line) of these simulated spectra was then able to approximate satisfactorily the experimentally observed shape of the signal for the $48+$ charge state of the modified protein (solid black line). 

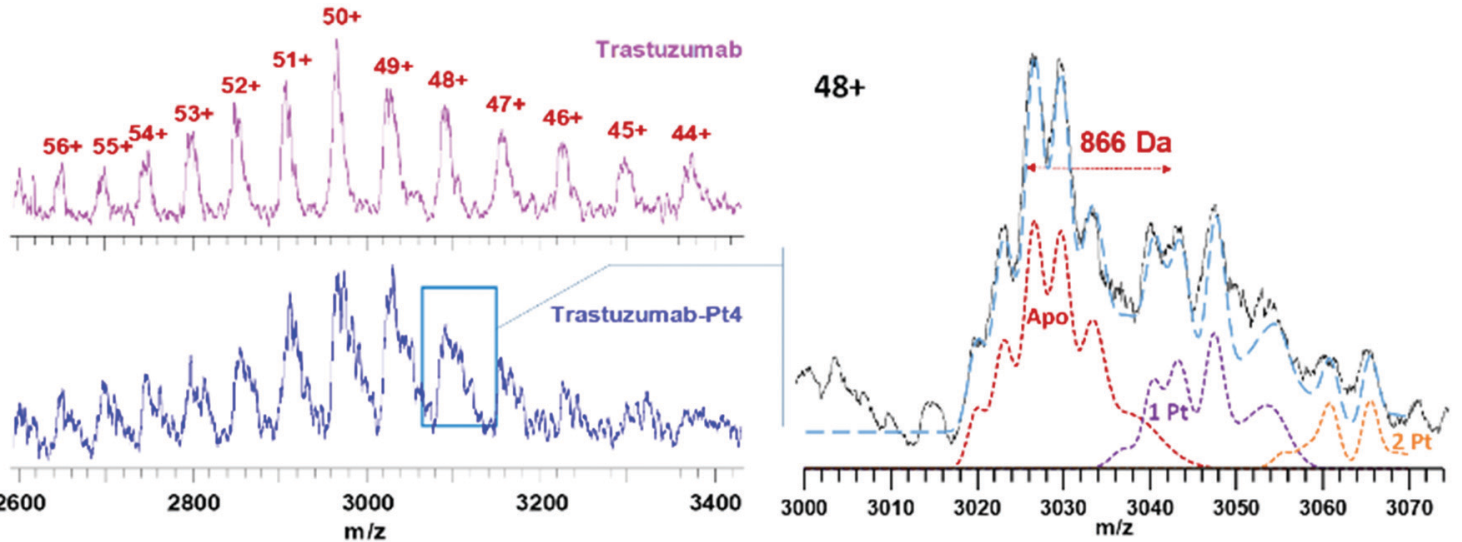

Fig. 4 Charge state distribution of trastuzumab before (top left) and after (bottom left) conjugation with Pt4. The panel on the right zooms in on the 48+ peak, showing the simulated spectra for trastuzumab with zero (Apo, red line), one Pt4 (purple line), and two Pt4 (orange line). Linear combination (blue line) of the three simulated spectra with relative intensities of $55: 30: 15$ is in agreement with the experimental data.

This analysis suggests that $c a .55 \%$ of the trastuzumab present was unmodified, while the adducts with 1 and 2 Pt4 per antibody represent 30 and $15 \%$ of the intensity, respectively, resulting in an average Pt4/trastuzumab ratio of $c a$. 0.6. This is in good agreement with the ratio obtained for the same batch by ICP-MS/UV-vis measurements $(\mathrm{Pt} /$ trastuzumab $=0.7)$. This lower degree of labelling for trastuzumab, compared to myoglobin, can be partially explained by considering the milder conjugation conditions employed in this case to avoid antibody denaturation (shorter conjugation time, lower $\mathrm{pH}$ ). In particular, isothiocyanate coupling is known to be very sensitive to $\mathrm{pH}$. While a lower Pt4/antibody ratio can be beneficial in this context, as it reduces heterogeneity, simplifying the immunoconjugate characterisation, higher $\mathbf{P t 4}$ /antibody ratios could be used to increase Pt4 delivery to cancer cells to maximise the biological effect. Immunoconjugates with higher Pt4/antibody ratio should be obtainable by increasing the molar equivalents of Pt4 used in the conjugation reaction as previously described. ${ }^{18}$

Overall, this work has shown that the new Pt(Iv) isothiocyanate derivative Pt4 undergoes facile conjugation to proteins and light-mediated release of its photoactivatable cytotoxic payload: prototype complex Pt1. Thus, Pt4 is a useful platform for protein-mediated delivery of photoactivatable Pt(Iv) agents. Our proof-of-concept conjugation experiment shows that Pt4 can conjugate to monoclonal antibodies under mild conditions, producing, for the first time, an antibody-drugconjugate containing a photoactivatable $\mathrm{Pt}(\mathrm{IV})$ agent.

This research was funded by the Wellcome Trust [209173/Z/ 17/Z] and EPSRC [EP/P030572/1]. For the purpose of open access, the author has applied a CC BY public copyright licence to any Author Accepted Manuscript version arising from this submission. F. L. acknowledges funding by the LOEWE project TRABITA funded by the Ministry of Higher Education, Research and the Arts (HMWK) of the State of Hesse.

\section{Conflicts of interest}

There are no conflicts to declare.

\section{Notes and references}

1 T. C. Johnstone, K. Suntharalingam and S. J. Lippard, Chem. Rev., 2016, 116, 3436-3486.

2 Y. R. Zheng, K. Suntharalingam, T. C. Johnstone, H. Yoo, W. Lin, J. G. Brooks and S. J. Lippard, J. Am. Chem. Soc., 2014, 136, 8790-8798.

3 J. Mayr, P. Heffeter, D. Groza, L. Galvez, G. Koellensperger, A. Roller, B. Alte, M. Haider, W. Berger, C. R. Kowol and B. K. Keppler, Chem. Sci., 2017, 8, 2241-2250.

4 V. Pichler, J. Mayr, P. Heffeter, O. Domotor, E. A. Enyedy, G. Hermann, D. Groza, G. Kollensperger, M. Galanksi, W. Berger, B. K. Keppler and C. R. Kowol, Chem. Commun., 2013, 49, 2249-2251.

5 D. P. McIntosh, R. J. Cooke, A. J. McLachlan, P. T. DaleyYates and M. Rowland, J. Pharm. Sci., 1997, 86, 1478-1483.

6 R. Huang, Y. Sun, Q. Gao, Q. Wang and B. Sun, Anti-Cancer Drugs, 2015, 26, 957-963.

7 R. Huang, Y. Sun, X.-Y. Zhang, B.-W. Sun, Q.-C. Wang and J. Zhu, Biomed. Pharmacother., 2015, 73, 116-122.

8 J. Ahn, Y. Miura, N. Yamada, T. Chida, X. Liu, A. Kim, R. Sato, R. Tsumura, Y. Koga, M. Yasunaga, N. Nishiyama, Y. Matsumura, H. Cabral and K. Kataoka, Biomaterials, 2015, 39, 23-30.

9 H. Y. Shi, C. Imberti and P. J. Sadler, Inorg. Chem. Front., 2019, 6, $1623-1638$.

10 H. Shi, Q. Wang, V. Venkatesh, G. Feng, L. S. Young, I. RomeroCanelón, M. Zeng and P. J. Sadler, Dalton Trans., 2019, 48, 8560-8564.

11 A. Gandioso, E. Shaili, A. Massaguer, G. Artigas, A. Gonzalez-Canto, J. A. Woods, P. J. Sadler and V. Marchan, Chem. Commun., 2015, 51, 9169-9172.

12 H. Y. Shi, C. Imberti, H. Y. Huang, I. Hands-Portman and P. J. Sadler, Chem. Commun., 2020, 56, 2320-2323.

13 R. Fraczkiewicz and W. Braun, J. Comput. Chem., 1998, 19, 319-333.

14 C. Imberti, P. Y. Zhang, H. Y. Huang and P. J. Sadler, Angew. Chem., Int. Ed., 2020, 59, 61-73.

15 https://www.accessdata.fda.gov/drugsatfda_docs/label/2010/103792s52 50lbl.pdf, 2021.

16 P. M. LoRusso, D. Weiss, E. Guardino, S. Girish and M. X. Sliwkowski, Clin. Cancer Res., 2011, 17, 6437-6447.

17 R. Upton, L. G. Migas, K. J. Pacholarz, R. G. Beniston, S. Estdale, D. Firth and P. E. Barran, Chem. Sci., 2019, 10, 2811-2820.

18 M. S. Cooper, E. Sabbah and S. J. Mather, Nat. Protoc., 2006, 1, 314-317. 